Hepatology. 2015 April ; 61(4): 1427-1429. doi:10.1002/hep.27676.

\title{
Prometheus and Progenitors
}

\author{
Anna Mae Diehl, MD \\ Department of Medicine, Duke University, Durham, North Carolina 27710
}

\section{Keywords}

Hippo; Yap; liver progenitors; ductular reaction

\section{Hippo pathway activity influences liver cell fate}

Yimlamai D, Christodoulou C, Galli GG, Yanger K, Pepe-Mooney B, Gurung B, Shrestha

K, Cahan P, Stanger BZ, Camargo FD

Cell 2014: 157:1324-38

PMID: 24906150

The Hippo-signaling pathway is an important regulator of cellular proliferation and organ size. However, little is known about the role of this cascade in the control of cell fate. Employing a combination of lineage tracing, clonal analysis, and organoid culture approaches, we demonstrate that Hippo pathway activity is essential for the maintenance of the differentiated hepatocyte state. Remarkably, acute inactivation of Hippo pathway signaling in vivo is sufficient to dedifferentiate, at very high efficiencies, adult hepatocytes into cells bearing progenitor characteristics. These hepatocyte-derived progenitor cells demonstrate self-renewal and engraftment capacity at the single-cell level. We also identify the NOTCH-signaling pathway as a functional important effector downstream of the Hippo transducer YAP. Our findings uncover a potent role for Hippo/YAP signaling in controlling liver cell fate and reveal an unprecedented level of phenotypic plasticity in mature hepatocytes, which has implications for the understanding and manipulation of liver regeneration.

The extraordinary regenerative capabilities of the liver have been recognized since the ancient Greeks. It has been presumed that the unique replicative ability of mature liver epithelial cells explains the liver's regenerative potential. Similar to other differentiated epithelial cells, adult hepatocytes and cholangiocytes are not proliferative during health. However, unlike other epithelial cell types, mature liver epithelial cells are able to re-enter the cell cycle. Following partial hepatectomy, for example, residual adult hepatocytes and cholangiocytes proliferate to regenerate resected liver mass. Thus, it was presumed that livers regenerate from other injuries mainly by reactivating mature liver epithelial cell

Corresponding Author: Anna Mae Diehl, MD, Division of Gastroenterology, Snyderman Building - Suite 1073, Duke University, Durham, North Carolina 27710, Phone: 919-684-2366 or 919-684-2616, annamae.diehl@dm.duke.edu. 
proliferation, and there was little motivation to search for adult liver progenitor compartments. Interest in liver progenitors was re-vitalized by growing evidence that functional liver mass is maintained despite inhibited proliferation of mature liver epithelial cells during various chronic liver diseases. Chronically injured livers accumulate various types of cells that are relatively inconspicuous in healthy adult livers, including small ovalshaped cells with a high nuclear/cytoplasmic ratio (oval cells) and epitheliod cells that cluster in primitive ductular structures (ductular-type progenitors). Both cell types tend to localize near cells that express myofibroblast markers in areas where extracellular matrix remodeling is active. This entire process has been dubbed the ductular reaction. (1) At any given point in time, the intensity of the ductular reaction generally correlates with the severity of liver fibrosis. (2) The ductular reaction also seems to be a pre-requisite for eventual liver regeneration because various interventions that prevent the response block recovery. (3) The latter observation prompted speculation that oval and/or ductular cells are liver progenitors. (4) This concept has been supported by other evidence that such cells are able to repopulate injured livers and regenerate healthy adult organs when transplanted into otherwise-fatal models of liver failure. (5)

The identity of the cell(s) of origin for these adult liver progenitors remains an open question. Possibilities include an extra-hepatic stem/progenitor cell (e.g., a bone marrowderived multi-potent progenitor), a liver resident multi-potent stem-like/progenitor cell, and one of the other adult liver cell types. Candidates in the latter category include adult hepatocytes, cholangiocytes, stellate cells, and endothelial cells. Solving this mystery has proven to be challenging and data have been published both supporting and refuting possible roles for each of these. (6) However, all of the findings are confounded by the limitations of currently available techniques, the inherent plasticity of the cell types of interest, and the importance of micro-environmental cues that control cell fate decisions in situ. In the current paper, Yimlamai and colleagues present evidence that adult hepatocytes are able to dedifferentiate and generate multi-potent liver progenitors. (7) Two general approaches were used to reach this conclusion. First the authors attempted to manipulate Hippo, a key liver growth-regulatory pathway, in cholangiocytes and found that this did not impact liver growth. Second, they showed that liver growth was significantly altered when Hippo pathway activity was manipulated in hepatocytes. Moreover, they demonstrated striking correlations between inhibition of Hippo signaling/nuclear accumulation of Yap in hepatocytes and the intensity of the subsequent ductular reaction which was comprised of immature Yap-positive cells harboring markers consistent with their derivation from hepatocytes. The Yap-positive immature cells were able to regenerate healthy liver tissue when transplanted into mice with massive acute liver injury.

Based on all of this evidence, the authors concluded that hepatocytes were likely to have been the resident adult liver cell type that generated the progenitor populations that emerged in these models. However, a few caveats merit consideration. First, it is not clear that the cytokeratin 19-targeting strategy used to manipulate Hippo signaling in cholangiocytes succeeded in achieving that objective in all of the various cholangiocyte subpopulations. If it did not, then it remains possible that certain types of ductular cells with progenitor capabilities were over-looked. This is particularly important because the authors 
demonstrated that nuclear accumulation of endogenous Yap, and endogenous Yap transcriptional activity, are largely restricted to the ductular compartment in adult mice and humans. Second, it is unclear if adult hepatocytes are uniformly (and persistently) capable of generating progenitors when Hippo signaling is altered experimentally. This point is particularly pertinent since these authors recently reported that tetraploidy inhibits Yap activation. Most hepatocytes in adult livers are polyploid and thus, would not be expected to activate endogenous Yap. Whether or not polyploidy influenced the constitutively active Yap transgene used in this study was not examined. Third, it is also known that the efficiency of adeno-associated virus (AAV)-mediated gene transfer to hepatocytes is significantly impacted by hepatic zonal heterogeneity. Age is also known to impact both the efficiency and persistence of $\underline{\mathrm{AAV}}$-directed gene transfer. In addition, the dose and duration of exposure to certain Cre-activating agents influences liver health. These factors might have influenced the efficiency/persistence of the adenovirus-directed, Cre-dependent gene manipulations in this study. Fourth, the authors acknowledge that their AAV-mediated targeting approach for hepatocytes also resulted in gene rearrangement in small numbers of immature ductular-type cells and oval-like cells. Since outcomes of the manipulation were examined weeks to months after AAV delivery, it is conceivable that rapid outgrowth/ differentiation of these other cell types might have contributed to the liver phenotypes that were ultimately observed. Fifth, whether or not the AAV approach resulted in gene transfer to sinusoidal cells (e.g., liver endothelial cells, hepatic stellate cells) was not examined directly. This is pertinent because in other tissues, Yap regulates the viability, proliferation, and differentiation of stem-like cells by controlling cell-cell and cell-matrix interactions that impact cell polarity and migration. Conversely, these interactions also control Yap activation. Stated another way, Hippo signaling regulates cell fate by modulating epithelialmesenchymal transitions (EMT), and EMT regulates Hippo pathway activity. (8) Adult hepatocytes (and mature ductular cells) are not thought to be capable of EMT in situ. However, both stellate cells and liver sinusoidal endothelial cell types readily transition to more mesenchymal phenotypes in injured livers. (9) Gene profiling of livers from mice in the present study demonstrated significant treatment-related differences in signaling pathways that are known to regulate EMT, including transforming growth factor beta, Notch, Wnt, and Hedgehog/smoothened. (7)

Despite the afore-mentioned considerations regarding which cell type(s) might have been responsible for the outcomes, the work clearly confirms the growing body of evidence that nuclear accumulation of active Yap in liver cells promotes liver growth/regeneration. In this study, Yap activation was mainly achieved by over-expressing a constitutively active Yap transgene. Therefore, questions remain regarding the signals that activate endogenous Yap during liver injury, and in which type(s) of liver cells this process occurs. It is also uncertain if Yap activation might have cell type-specific consequences and if so, how these differential Yap effects might interact to orchestrate liver growth. Research to address these gaps in knowledge is underway. For example, toxic levels of bile acids were recently shown to activate Yap and trigger the ductular reaction in mice with cholestatic liver injury caused by bile duct ligation. Nuclear localization of Yap was also demonstrated in ductular cells in different types of fibrosing cholestatic disease in humans, including congenital hepatic fibrosis and extrahepatic biliary atresia. (10) In the studies reported by Yimlamai al, Yap 
activation was found to induce Notch signaling, thereby uncovering novel mechanisms that are likely to influence both reconstruction of the biliary tree and liver metabolism in regenerating livers. (7) Prometheus would be comforted by the accumulating evidence that adult livers can exploit multiple strategies to assure recovery from liver damage.

\section{References}

1. Theise ND, Saxena R, Portmann BC, Thung SN, Yee H, Chiriboga L, Kumar A, et al. The canals of Hering and hepatic stem cells in humans. Hepatology. 1999; 30:1425-1433. [PubMed: 10573521]

2. Williams MJ, Clouston AD, Forbes SJ. Links between hepatic fibrosis, ductular reaction, and progenitor cell expansion. Gastroenterology. 2014; 146:349-356. [PubMed: 24315991]

3. Tirnitz-Parker JE, Viebahn CS, Jakubowski A, Klopcic BR, Olynyk JK, Yeoh GC, Knight B. Tumor necrosis factor-like weak inducer of apoptosis is a mitogen for liver progenitor cells. Hepatology. 2010; 52:291-302. [PubMed: 20578156]

4. Yoon SM, Gerasimidou D, Kuwahara R, Hytiroglou P, Yoo JE, Park YN, Theise ND. Epithelial cell adhesion molecule (EpCAM) marks hepatocytes newly derived from stem/progenitor cells in humans. Hepatology. 2011; 53:964-973. [PubMed: 21319194]

5. Li WL, Su J, Yao YC, Tao XR, Yan YB, Yu HY, Wang XM, et al. Isolation and characterization of bipotent liver progenitor cells from adult mouse. Stem Cells. 2006; 24:322-332. [PubMed: 16109753]

6. Shin S, Kaestner KH. The origin, biology, and therapeutic potential of facultative adult hepatic progenitor cells. Curr Top Dev Biol. 2014; 107:269-292. [PubMed: 24439810]

7. Yimlamai D, Christodoulou C, Galli GG, Yanger K, Pepe-Mooney B, Gurung B, Shrestha K, et al. Hippo pathway activity influences liver cell fate. Cell. 2014; 157:1324-1338. [PubMed: 24906150]

8. Varelas X, Samavarchi-Tehrani P, Narimatsu M, Weiss A, Cockburn K, Larsen BG, Rossant J, et al. The Crumbs complex couples cell density sensing to Hippo-dependent control of the TGF-betaSMAD pathway. Dev Cell. 2010; 19:831-844. [PubMed: 21145499]

9. Xie G, Diehl AM. Evidence for and against epithelial-to-mesenchymal transition in the liver. Am J Physiol Gastrointest Liver Physiol. 2013; 305:G881-890. [PubMed: 24157970]

10. Anakk S, Bhosale M, Schmidt VA, Johnson RL, Finegold MJ, Moore DD. Bile acids activate YAP to promote liver carcinogenesis. Cell Rep. 2013; 5:1060-1069. [PubMed: 24268772] 